\title{
Scaling Laws for Photoelectron Holography in the Midinfrared Wavelength Regime
}

\author{
Y. Huismans, ${ }^{1}$ A. Gijsbertsen, ${ }^{1}$ A. S. Smolkowska, ${ }^{1}$ J. H. Jungmann, ${ }^{1}$ A. Rouzée,,${ }^{1,2}$ P. S. W. M. Logman, ${ }^{1}$ F. Lépine, ${ }^{3}$ \\ C. Cauchy, ${ }^{3}$ S. Zamith, ${ }^{4}$ T. Marchenko, ${ }^{5}$ J. M. Bakker, ${ }^{6}$ G. Berden, ${ }^{6}$ B. Redlich, ${ }^{6}$ A. F. G. van der Meer, ${ }^{6}$ M. Yu. Ivanov, \\ T.-M. Yan, ${ }^{8,9}$ D. Bauer, ${ }^{8}$ O. Smirnova, ${ }^{2}$ and M. J. J. Vrakking ${ }^{1,2}$ \\ ${ }^{1}$ FOM-Institute AMOLF, Science Park 104, 1098 XG Amsterdam, Netherlands \\ ${ }^{2}$ Max-Born-Institut, Max Born Straße 2A, D-12489 Berlin, Germany \\ ${ }^{3}$ Université Lyon I, CNRS, LASIM, UMR 5579, Bâtiment Kastler, 43, Boulevard du 11 Novembre 1918 , \\ F69622 Villeurbanne Cedex, France \\ ${ }^{4}$ Laboratoire Collisions, Agrégats, Réactivité, IRSAMC, UPS, Université de Toulouse and UMR 5589 CNRS, 31062 Toulouse, France \\ ${ }^{5}$ UPMC Université Paris 06, CNRS, UMR 7614, Laboratoire de Chimie Physique Matière et Rayonnement, F-75005 Paris, France \\ ${ }^{6}$ FOM-Institute for Plasma Physics Rijnhuizen, Edisonbaan 14, 3439 MN Nieuwegein, Netherlands \\ ${ }^{7}$ Imperial College, London SW7 2BW, United Kingdom \\ ${ }^{8}$ Institut für Physik, Universität Rostock, 18051 Rostock, Germany \\ ${ }^{9}$ Max-Planck-Institut für Kernphysik, 69029 Heidelberg, Germany
}

(Received 21 September 2011; revised manuscript received 18 January 2012; published 6 July 2012)

\begin{abstract}
Midinfrared strong-field laser ionization offers the promise of measuring holograms of atoms and molecules, which contain both spatial and temporal information of the ion and the photoelectron with subfemtosecond temporal and angstrom spatial resolution. We report on the scaling of photoelectron holographic interference patterns with the laser pulse duration, wavelength, and intensity. High-resolution holograms for the ionization of metastable xenon atoms by 7-16 $\mu \mathrm{m}$ light from the FELICE free electron laser are presented and compared to semiclassical calculations that provide analytical insight.
\end{abstract}

DOI: 10.1103/PhysRevLett.109.013002

PACS numbers: $32.80 . \mathrm{Rm}, 32.80 . \mathrm{Wr}$

The concept of holography [1] can be applied to strong-field laser ionization to record temporal and spatial information on the atomic and molecular scale [2]. In conventional holography, a coherent beam of light or electrons is split into a signal and a reference beam. The signal beam scatters off the target and, upon recombination with the reference beam, creates an interference pattern that stores spatial information. In strong-field photoelectron holography (SFPH), the electron beam is created by laser-induced tunnel ionization. After tunneling, the electron wave packet moves in the oscillatory laser field and can follow two paths en route to the detector. For small orthogonal velocities with respect to the laser polarization, the electron is driven back to the ion where it can scatter off, generating a signal wave. For large orthogonal velocities, the electron makes a wide turn around the ion and forms a reference wave. The interference of the signal and reference waves on a detector creates the photoelectron hologram. The benefits of using SFPH are twofold. First, very high electron densities can be obtained at the ionic target [3]. Second, spatial and temporal information are encoded for both the target ion and the electron [4]. With midinfrared lasers, femtosecond and attosecond resolution can be obtained for, respectively, the ion and electron dynamics.

SFPH may be regarded as being complementary to another photoelectron holography method being developed in gas phase molecular physics [5]. Building on a wellestablished approach in solid state physics [6], ionization with extreme ultraviolet or x-ray light can create photoelectrons with a Broglie wavelength comparable to the interatomic distances in molecules, which can go to the detector directly or scatter off one of the neighboring atoms, and resulting in an interference pattern (in the molecular frame) that constitutes a hologram [7]. Using intense and short pulsed x-ray sources, like the Lineac Coherent Light Source [8], x-ray induced photoelectron holograms can be measured that allow visualizing, with femtosecond time resolution, atomic motion in molecules undergoing dynamics. The advantage of SFPH is that the required high energy photoelectrons are efficiently generated by midinfrared strong-field ionization, implying that, using standard nonlinear optical techniques, a tabletop experiment can be performed. Furthermore, the SFPH hologram can record temporal and spatial information on molecules, as well as - with attosecond time resolutiondynamics of the photoelectron, since the electron motion is guided by an oscillatory laser field.

To better understand SFPH, we explore how the holograms scale with laser intensity $I_{\text {laser }}$, wavelength $\lambda_{\text {laser }}$, and pulse duration $\tau_{\text {laser }}$. Experiments were carried out at the free electron laser for intracavity experiments (FELICE), where metastable xenon atoms $\left[5 p^{5}\left({ }^{2} P_{3 / 2}\right) 6 s[3 / 2]_{2}\right]$ were ionized using 5-20 cycle mid-IR laser pulses with wavelengths ranging from 7 to $16 \mu \mathrm{m}$. The metastable xenon atoms (produced by electron impact excitation [9]) were introduced into an experimental apparatus that was integrated into the FELICE laser cavity, and ionized by the free 
electron laser (FEL). In the velocity map imaging (VMI) detector, the photoelectrons were accelerated towards a position-sensitive detector [10] consisting of a dual microchannel plate, a phosphor screen, and a CCD camera. The laser polarization was parallel to the imaging detector, allowing a reconstruction of the $3 \mathrm{D}$ velocity distribution by an Abel inversion routine. The wavelength and pulse duration were controlled by changes to the FEL operation, whereas the intensity was varied by moving the experimental apparatus along the laser propagation direction.

In what follows, the experimental results will be compared to two semiclassical methods that are based on the strong field approximation (SFA) [11-13]. In standard SFA, Coulomb forces on the electron are assumed negligibly small after tunnel ionization, ruling out scattering of the electron wave packet upon returning to the target ion. In SFPH, however, electron-ion scattering is crucial, and therefore in the generalized SFA (gSFA) method scattering is included by assuming that an electron that returns to the core with momentum $\mathbf{k}$, scatters to a momentum $\mathbf{p}$ (with $|\mathbf{p}|=|\mathbf{k}|)$.

Within this model, the final wave packet that reaches the detector can be expressed as

$$
\psi=\psi_{\text {signal }}+\psi_{\text {ref }}
$$

Whereas the signal wave packet $\psi_{\text {signal }}$ scatters off the target, the reference wave packet $\psi_{\text {ref }}$ only experiences the influence of the laser field. The phase difference between the two wave packets is [2]

$$
\begin{aligned}
\Delta \phi= & \phi_{\text {signal }}-\phi_{\text {ref }} \\
= & -\frac{1}{2} \int_{t_{0}^{\text {signal }}}^{t_{C}} v_{z}^{2} d \tau+\frac{1}{2} \int_{t_{0}^{\text {ref }}}^{t_{C}} v_{z}^{2} d \tau-\frac{1}{2} p_{r}^{2}\left(t_{C}-t_{0}^{\text {ref }}\right) \\
& +I P \Delta t_{0}+\Delta S^{\mathrm{Im}}
\end{aligned}
$$

with the phase of the signal and reference wave packet defined as $\phi_{\text {signal }}$ and $\phi_{\text {ref }}$. On the right side of this equation the real part of the ionization times of, respectively, the reference and signal wave packet are $t_{0}^{\text {ref }}$ and $t_{0}^{\text {signal }}$, as defined by the saddle point method [14]. The difference in ionization times is $\Delta t_{0}$ and the recollision time is $t_{C}$. The velocity of the wave packet along the laser polarization is $v_{z}$ and its final orthogonal momentum is $p_{r} . I P$ is the ionization potential and $\Delta S^{\mathrm{Im}}$ is the difference in action in imaginary time. For a better understanding we interpret each term. The first and second term represent the phase evolution of the signal and reference wave parallel to the laser polarization, and the third term is the phase difference acquired in the orthogonal direction. The fourth term is caused by a difference in the ionization times of the reference and signal waves, and the last term is the phase difference acquired during propagation in imaginary time (i.e., during the tunnel ionization). The third term in expression (2) has been identified as the key term responsible for the formation of the hologram [2], i.e.,

$$
\Delta \phi \approx-\frac{1}{2} p_{r}^{2}\left(t_{C}-t_{0}^{\mathrm{ref}}\right) .
$$

A more detailed description is given in the supporting online material (SOM) of Ref. [2].

Whereas the gSFA method assumes short-range scattering, the Coulomb corrected strong field approximation (CCSFA) method $[2,15,16]$ corrects both the signal and reference electron trajectories for the long-range Coulomb force. Saddle point times are calculated according to the standard SFA method [14], i.e., neglecting the Coulomb force, and providing the initial conditions for complex electron trajectories that are propagated under the influence of both the Coulomb and laser field. A detailed description is given in Refs. [2,15]. Unless mentioned otherwise, the CCSFA calculations presented below are results for ionization from a single cycle out of a flattop laser pulse.

Figure 1 reports a series of measurements using $7 \mu \mathrm{m}$ FEL radiation. By moving the spectrometer along the laser
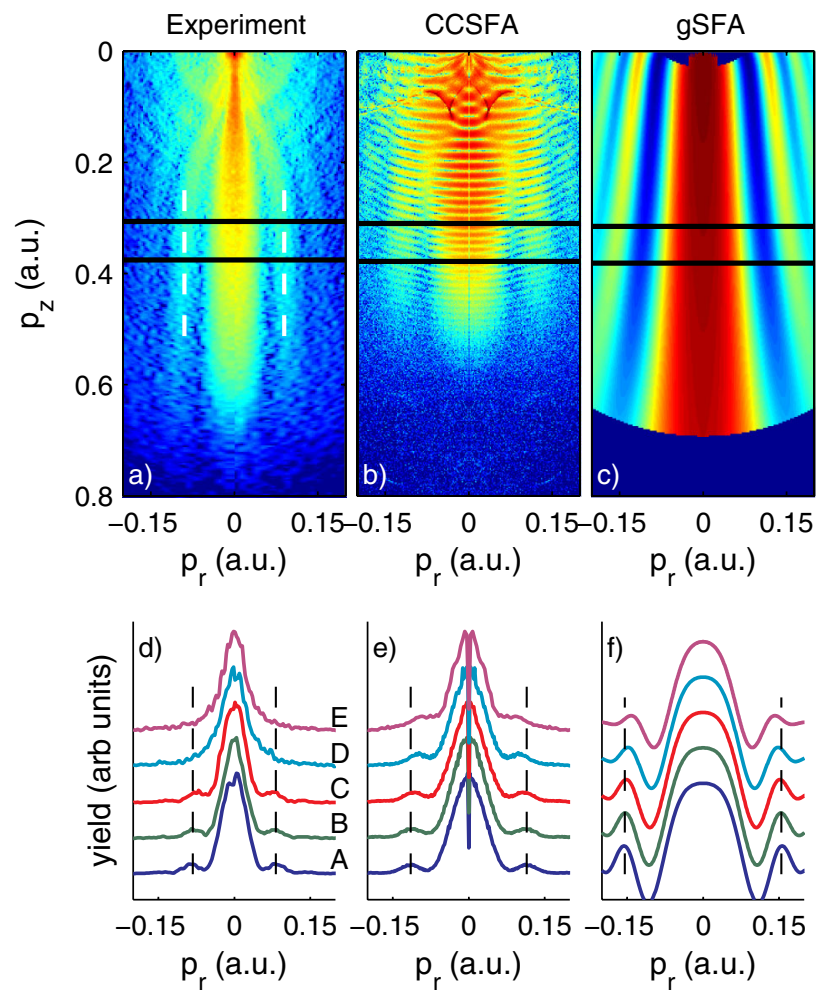

FIG. 1 (color online). Scaling of photoelectron holography with intensity. The top panel gives momentum maps for, respectively, the experimental data, CCSFA and gSFA calculations at $\lambda_{\text {laser }}=7 \mu \mathrm{m}$ and $I=7.1 \times 10^{11} \mathrm{~W} / \mathrm{cm}^{2}$. The bottom panel shows lineouts taken at constant $p_{z}$ for different intensities: (A) $I_{\text {laser }}=7.1 \times 10^{11} \mathrm{~W} / \mathrm{cm}^{2},(B) I_{\text {laser }}=5.5 \times 10^{11} \mathrm{~W} / \mathrm{cm}^{2}$, (C) $I_{\text {laser }}=4.5 \times 10^{11} \mathrm{~W} / \mathrm{cm}^{2}$, (D) $I_{\text {laser }}=3.2 \times 10^{11} \mathrm{~W} / \mathrm{cm}^{2}$, (E) $I_{\text {laser }}=1.9 \times 10^{11} \mathrm{~W} / \mathrm{cm}^{2}$. The lineouts are marked by black lines in the figures in the top panel, and are taken at $\left[0.5 p_{z}^{\text {cutoff }}\right]$, where the $p_{z}^{\text {cutoff }}$ corresponds to the $2 U p$ cutoff in energy. For the conditions shown in the top panel, $2 U p$ is at a momentum of approximately 0.7 a.u. 
propagation axis, $I_{\text {laser }}$ could be varied between $1.9 \times$ $10^{11} \mathrm{~W} / \mathrm{cm}^{2}$ and $7.1 \times 10^{11} \mathrm{~W} / \mathrm{cm}^{2}$. The maximum energy an electron can acquire without scattering is $2 U_{p}$ [17], with $U_{p}(\mathrm{eV})=9.33 \times 10^{-14} I_{\text {laser }}\left[\mathrm{W} / \mathrm{cm}^{2}\right] \lambda_{\text {laser }}{ }^{2}[\mu \mathrm{m}]$, thus allowing retrieval of $I_{\text {laser }}$ from the observed experimental cutoff. In Fig. 1(a), the experimentally obtained momentum map is shown for the highest intensity. The image shows a dominant photoelectron emission along the laser polarization, and well-resolved sidelobes, marked by white dashed lines. These sidelobes are identified as holographic interference structures and are well reproduced by the CCSFA and gSFA calculations [Figs. 1(b) and 1(c)]. The CCSFA method quantitatively reproduces the fringe spacing and shape, whereas the gSFA calculation gives only qualitative agreement, as a result of neglecting the long-range Coulomb interaction. Furthermore, in the gSFA calculations the fringes widen towards higher parallel
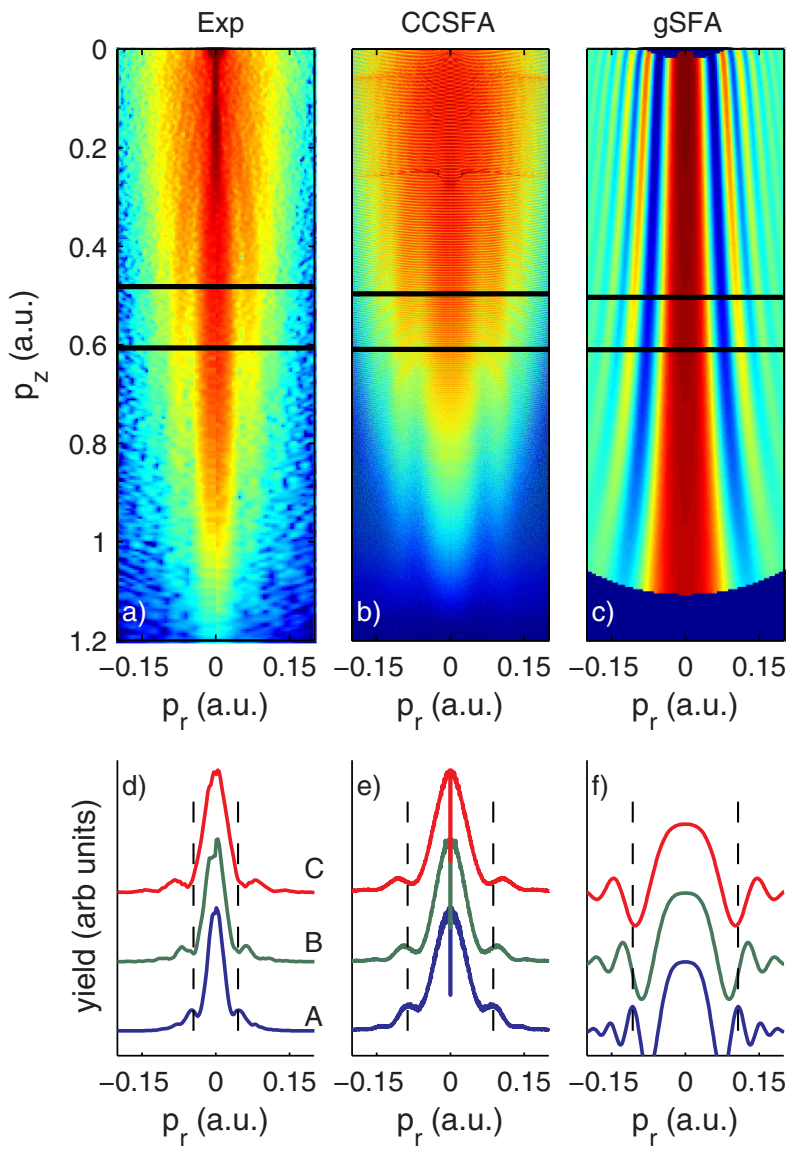

FIG. 2 (color online). Scaling of photoelectron holography with wavelength. The top panel gives momentum maps for, respectively, the experimental data, CCSFA, and gSFA calculations at $\lambda_{\text {laser }}=16 \mu \mathrm{m}$ and $I_{\text {laser }}=3.4 \times 10^{11} \mathrm{~W} / \mathrm{cm}^{2}$. The bottom panel shows lineouts taken at constant $p_{z}$ for (A) $\lambda_{\text {laser }}=16 \mu \mathrm{m}$ and $I_{\text {laser }}=3.4 \times 10^{11} \mathrm{~W} / \mathrm{cm}^{2}$, (B) $\quad \lambda_{\text {laser }}=11 \mu \mathrm{m}$ and $I_{\text {laser }}=4.4 \times 10^{11} \mathrm{~W} / \mathrm{cm}^{2}$, and (C) $\lambda_{\text {laser }}=8 \mu \mathrm{m}$ and $I_{\text {laser }}=5.4 \times 10^{11} \mathrm{~W} / \mathrm{cm}^{2}$. The lineouts are taken at $\left[0.5 p_{z}^{\text {cutoff }}\right]$. momenta, while in the experiment and in the CCSFA the fringes are parallel to the polarization axis at high momentum. The black lines in the momentum maps mark areas where a series of lineouts are taken [Figs. 1(d)-1(f)]. Decreasing the intensity from $7.1 \times 10^{11} \mathrm{~W} / \mathrm{cm}^{2}$ to $4.5 \times$ $10^{11} \mathrm{~W} / \mathrm{cm}^{2}$ leaves the fringe spacing virtually unchanged. At lower intensities the fringes are not resolved anymore in the experimental data, though the CCSFA and gSFA calculations show that for the lower intensities the fringe spacing starts narrowing.

A wavelength scan is presented in Fig. 2. In Fig. 2(a) a momentum map is shown for ionization of metastable xenon atoms by $16 \mu \mathrm{m}$ light. Again good quantitative agreement is obtained with the CCSFA method [Fig. 2(b)], and only qualitative agreement with the gSFA method [Fig. 2(c)]. As the lineouts in Figs. 2(d)-2(f) show, upon changing the wavelength from 16 to $8 \mu \mathrm{m}$, the fringe spacing clearly increases.

In the experiment the laser pulse duration could not be changed in a well-controlled manner. Therefore the evolution of the interference fringes with pulse duration is only investigated numerically using the CCSFA method, and using realistic sine-squared pulses (Fig. 3). The fringe spacing does not change with the pulse duration.

According to Figs. 1-3, the fringe spacing is independent of the laser pulse duration, changes slightly with intensity, and changes significantly as a function of
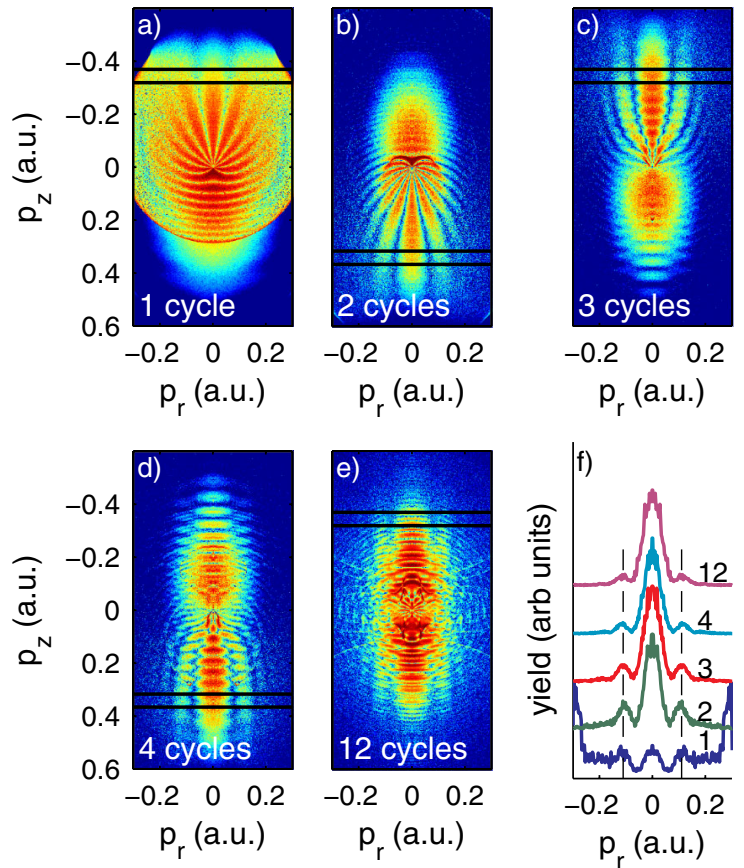

FIG. 3 (color online). Scaling of photoelectron holography with pulse duration. The five momentum maps are CCSFA calculations for $\lambda_{\text {laser }}=7 \mu \mathrm{m}, I_{\text {laser }}=7.1 \times 10^{11} \mathrm{~W} / \mathrm{cm}^{2}$, and a different number of laser cycles with a sine-square envelope of the laser pulse. The lineouts are displayed in the bottom right figure and are taken at $\left[0.5 p_{z}^{\text {cutoff }}\right]$. 
wavelength. We analyze this behavior using Eq. (3), which suggests that the interference pattern scales with $\Delta t=t_{C}-t_{0}^{\text {ref }}$. This time difference is largely caused by the time the electron spends in the continuum [Fig. 4(a)]. In the high intensity regime $\Delta t$ is almost intensity independent, since the birth $\left(t_{B}\right)$ and return times $\left(t_{R}\right)$ stay the same. The intensity-dependent effect is associated with the relatively small (compared to the oscillation amplitude) displacement of the electron from the origin upon tunneling $\Delta x$, which increases the time difference from $t_{R}$ to $t_{C}$ by an amount $\Delta x / v\left(t_{R}\right)$. In the high intensity regime $(\gamma \ll 1$, where $\gamma=\sqrt{I P / 2 U P}$ is the Keldysh parameter) one can easily check that this extra time scales as $\gamma^{2}$, while in the low intensity regime $(\gamma \gg 1)$ it scales with $\gamma$. Consequently, this contribution is low for high intensities $(\gamma \ll 1)$ and no substantial scaling with intensity is expected in this regime. For lower intensities the extra time becomes substantial and a modest narrowing of the fringe spacing is expected [Fig. 4(b)]. To support these conclusions, the cosine of the approximate reference-versussignal phase difference, as given by Eq. (3) and that
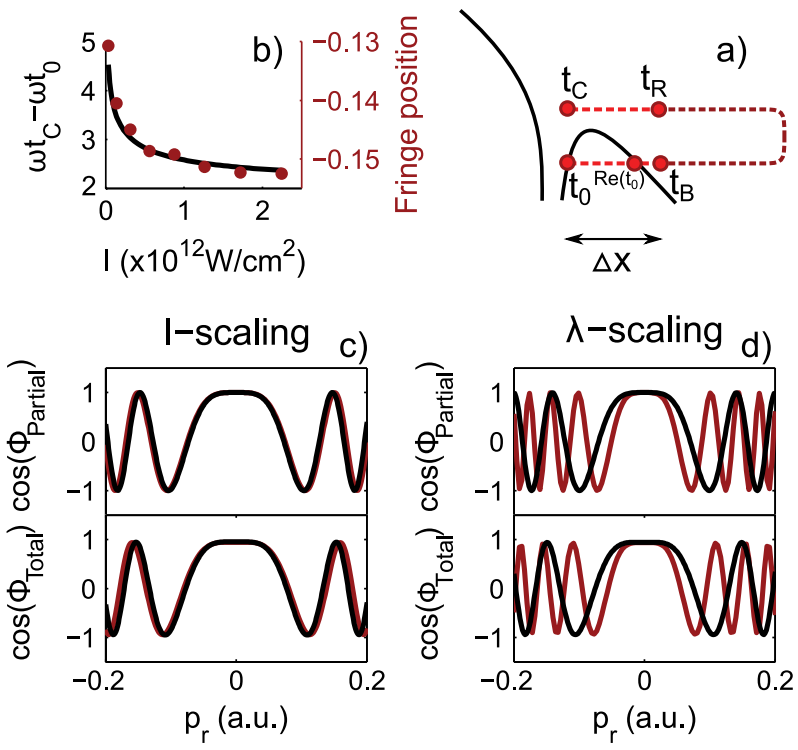

FIG. 4 (color online). gSFA calculations for photoelectron holography. (a) Tunneling and evolution of an electron wave packet in a laser field, illustrating the time the electron enters the barrier $\left(t_{0}\right)$, exits the barier $\left[\operatorname{Re}\left(t_{0}\right)\right]$, has zero parallel velocity $\left(t_{B}\right)$, returns to the point of zero parallel velocity $\left(t_{R}\right)$, and scatters $\left(t_{C}\right)$. (b) scaling with intensity of the difference $\omega \Delta t=$ $\omega\left(t_{C}-t_{0}\right)$ for $\omega=0.0065$ a.u. $\left(\lambda_{\text {laser }}=7 \mu \mathrm{m}\right)$ (black line). The phase difference $\omega \Delta t$ is taken for a birth time of the electron at a phase of $0.3 \pi$ in $A=A_{0} \sin \left(\omega t_{B}\right)$. With increasing intensity, $\omega \Delta t$ reaches an asymptotic value. In red dots the fringe positions are shown, which depend on $\omega \Delta t$. (c) The cosine of the total phase difference $\left(\phi_{\text {total }}\right)$ and the approximated phase difference $\left(\phi_{\text {partial }}\right)$ as described by Eqs. (2) and (3) in the text, for intensities of $7.1 \times 10^{11} \mathrm{~W} / \mathrm{cm}^{2}$ (red) and $3.6 \times 10^{11} \mathrm{~W} / \mathrm{cm}^{2}$ (black). (d) Same as figure (c), but now for different wavelengths; $8 \mu \mathrm{m}$ (black) and $16 \mu \mathrm{m}$ (red). of the full phase difference as given by Eq. (2) are compared at a wavelength of $7 \mu \mathrm{m}$ and for two intensities in Fig. 4(c). The weak dependence of the fringe spacing on the intensity explains why the hologram is visible in experiments where a range of intensities in the focus are sampled.

If instead the wavelength is varied, $\Delta t$ does change. When the wavelength is doubled, the time between ionization and recollision is doubled and consequently the cosine of the phase difference changes twice as fast. With this, the fringe spacing narrows, as shown in Fig. 4(d), where the cosine of the approximate and total phase difference are shown for wavelengths of 8 and $16 \mu \mathrm{m}$ and an intensity of $7.1 \times 10^{11} \mathrm{~W} / \mathrm{cm}^{2}$. Again the approximate phase difference deviates marginally from the full phase difference, justifying the approximation made. The evolution with wavelength shown is in agreement with the experimental and theoretical observations in Fig. 2.

No significant changes are observed when the pulse duration is varied, except for the appearance of other interference structures that become more prominent when the number of cycles increases. Since the electron wave packets that form the hologram are generated within one and the same quarter cycle, no changes in the fringe spacing are expected.

In conclusion, we have demonstrated that SFPH holograms are clearly visible for a wide range of parameters in the mid-IR wavelength regime, and can be well reproduced with the CCSFA method. The simple and fast gSFA method, which ignores the Coulomb tail along the trajectory, qualitatively predicts the scaling of the fringe spacing with intensity and wavelength. More sophisticated models like an improved gSFA model that includes the long-range Coulomb interaction will be needed to retrieve the spatial and temporal information that is stored in the hologram.

This information is potentially very valuable, and may allow the development of novel probes of atomic and molecular dynamics. A logical extension, for example, would be to perform SFPH experiments for aligned molecules, and to attempt retrieval of the molecular structure [18]. Subsequently SFPH could be used to time resolve molecular dynamics without having to rely heavily, as is presently often the case, on preexisting knowledge about the structural dependence of the molecular absorption spectrum. To do so, it will be of interest to scale the laser intensity and wavelength to approximately $10^{14} \mathrm{~W} / \mathrm{cm}^{2}$ and $3 \mu \mathrm{m}$, respectively, so that (i) the ionization of groundstate orbitals of small molecules becomes possible, and (ii) photoelectrons with several hundred $\mathrm{eV}$ of kinetic energy are produced, which can diffract off and encode the molecular structure.

This work is part of the research program of the "Stichting voor Fundamenteel Onderzoek der Materie (FOM)," which is financially supported by the "Nederlandse organisatie voor Wetenschappelijk 
Onderzoek (NWO)." M. Ivanov is supported by Science and Innovation Grant No. EP/E036112/1 of the Engineering and Physical Sciences Research Council. Financial support of the ATTOFEL ITN is gratefully acknowledged. T.-M. Yan is supported by the IMPRSQD, Heidelberg. D. Bauer is supported by the SFB 652 of the German Science Foundation. We thank S. V. Popruzhenko for his valuable contribution to the CCSFA-method.

[1] D. Gabor, Nobel Lectures, Physics (World Scientific, Singapore, 1992), pp. 1971-1980.

[2] Y. Huismans et al., Science 331, 61 (2011).

[3] H. Niikura, F. Légaré, R. Hasbani, A. D. Bandrauk, M. Yu. Ivanov, D. M. Villeneuve, and P. B. Corkum, Nature (London) 417, 917 (2002).

[4] M. Spanner, O. Smirnova, P. B. Corkum, and M. Yu Ivanov, J. Phys. B 37, L243 (2004).

[5] F. Krasniqi, B. Najjari, L. Strüder, D. Rolles, A. Voitkiv, and J. Ullrich, Phys. Rev. A 81, 033411 (2010).

[6] J. J. Barton, Phys. Rev. Lett. 61, 1356 (1988).
[7] A. Landers et al., Phys. Rev. Lett. 87, 013002 (2001).

[8] P. Emma et al., Nature Photon. 4, 641 (2010).

[9] A. Kohlhase and S. Kita, Rev. Sci. Instrum. 57, 2925 (1986).

[10] A. T. J. B. Eppink and D. H. Parker, Rev. Sci. Instrum. 68, 3477 (1997).

[11] L. V. Keldysh, Zh. Eksp. Teor. Fiz. 47, 1945 (1964) [Sov. Phys. JETP 20, 1307 (1965)].

[12] F. H. M. Faisal, J. Phys. B 6, L89 (1973).

[13] H. R. Reiss, Phys. Rev. A 22, 1786 (1980).

[14] R. Kopold, W. Becker, and M. Kleber, Opt. Commun. 179, 39 (2000).

[15] S. V. Popruzhenko and D. Bauer, J. Mod. Opt. 55, 2573 (2008).

[16] T.-M. Yan, S. V. Popruzhenko, M. J. J. Vrakking, and D. Bauer, Phys. Rev. Lett. 105, 253002 (2010).

[17] H. B. van Linden van den Heuvell and H. G. Muller, in Cambridge Studies in Modern Optics (Cambridge University Press, Cambridge, 1988), Vol. 8, p. 25.

[18] M. Peters, T. T. Nguyen-Dang, C. Cornaggia, S. Saugout, E. Charron, A. Keller, and O. Atabek, Phys. Rev. A 83, 051403 (2011). 